\title{
Cardiomiopatia Induzida por Estresse: Diagnóstico Diferencial de Infarto do Miocárdio nas Emergências
}

\author{
Renne Busnello ${ }^{1}$
}

\section{RESUMO}

A cardiomiopatia induzida por estresse pode mimetizar o diagnóstico de infarto agudo do miocárdio (IAM) nas salas de emergências. Este caso descreve uma paciente com quadro clínico de IAM. Após ser submetida a coronariografia, foi modificado seu diagnóstico para cardiomiopatia induzida por estresse. Seu conhecimento é importante, visto que é uma síndrome com características, tratamento e prognóstico diferentes do IAM.

DESCRITORES: Cardiomiopatia de Takotsubo. Disfunção ventricular esquerda. Estresse. Estresse psicológico/complicações.
C ardiomiopatia induzida por estresse é uma síndrome composta por dor torácica, alterações eletrocardiográficas e enzimáticas que mimetizam o infarto agudo do miocárdio, associada a disfunção do segmento apical do ventrículo esquerdo transitório e, menos frequentemente, do segmento médio do ventrículo esquerdo, porém na ausência de doença arterial angiográfica significativa ${ }^{1-7}$.

A cardiomiopatia induzida por estresse, também conhecida por cardiomiopatia de Takotsubo, foi descrita inicialmente no Japão por Satoh et al. ${ }^{1}$. O nome deriva da forma de balão do ventrículo esquerdo, que é similar à armadilha de polvo utilizada no Japão (takotsubo, em japonês). A anormalidade mais comum é a hipocinesia segmentar apical do ventrículo esquerdo transitória associada a hipercinesia compensatória na parede basal, produzindo a forma típica de balão durante a sístole. Menos frequentemente ocorre hipocinesia transitória mais evidente do segmento médio do ventrículo esquerdo (cerca de $40 \%$ dos casos), configurando uma

\footnotetext{
${ }^{1}$ Hospital Nossa Senhora da Conceição - Porto Alegre, RS, Brasil. Correspondência: Renne Busnello. Rua Roque Calage, 870/701 Passo da Areia - Porto Alegre, RS, Brasil - CEP 91350-090 E-mail: rennegb@cardiol.br

Recebido em: 16/1/2009 - Aceito em: 16/4/2009
}

\section{ABSTRACT}

Stress Cardiomyopathy: Differential Diagnosis of Myocardial Infarction in the Emergency Room

Stress cardiomyopathy may mimic the diagnosis of acute myocardial infarction (AMI) in emergency rooms. This paper describes a female patient with clinical AMI symptoms. After she was submitted to coronary angiography, the diagnosis was changed to stress cardiomyopathy. It is therefore important to acknowledge this case because the characteristics, treatment and prognosis of this syndrome are different from those of an AMI.

DESCRIPTORS: Takotsubo cardiomyopathy. Ventricular dysfunction, left. Stress. Stress, psychological/complications.

forma atípica de cardiomiopatia induzida por estresse. Recentemente, essa doença tem sido descrita em países ocidentais $^{5,6}$.

Este relato de caso tem o objetivo de divulgar o diagnóstico da cardiomiopatia induzida por estresse, visto que é uma doença que pode mimetizar o infarto agudo do miocárdio em até $2 \%$ dos casos atendidos nas emergências.

\section{RELATO DO CASO}

Paciente do sexo feminino, 50 anos, casada, pósmenopausa, sedentária, foi admitida na emergência com dor torácica anginosa de duração maior que 20 minutos, associada a sudorese e vômito. Não havia relato de doenças prévias ou uso de medicações. Não fumava. Havia sido resgatada de um incêndio. Sinais vitais na chegada eram pressão arterial de 130/60 mmHg, frequência cardíaca de 84 bpm e frequência respiratória de $20 \mathrm{rpm}$. Saturação arterial de oxigênio de 96\% em ar ambiente. Auscultas cardíaca e respiratória normais. Eletrocardiograma de 12 derivações evidenciava inversão da onda T nas regiões ântero-lateral e lateral alta (Figura 1).

A radiografia de tórax em posição ântero-posterior evidenciava cardiomegalia e discreto infiltrado intersticial bilateral em bases. Foi solicitada dosagem dos biomarcadores cardíacos - troponina T, 0,16 ng/dl e 0,20 ng/dl 


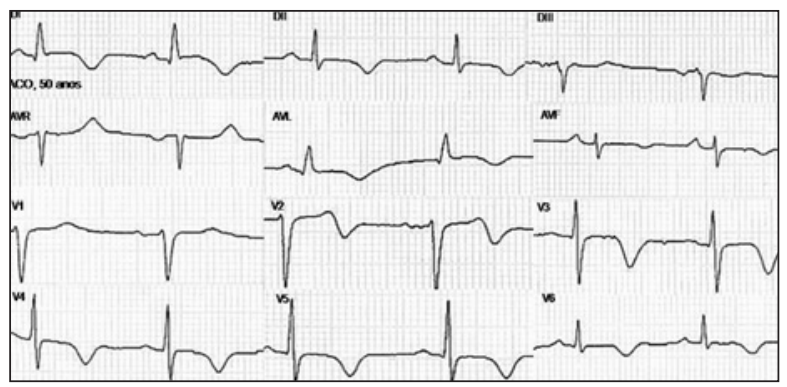

Figura 1 - Eletrocardiograma de 12 derivações evidenciando inversão da onda $\mathrm{T}$ em parede ântero-lateral e lateral alta.

(normal $<0,01 \mathrm{ng} / \mathrm{dl}$ ), e creatina quinase fração $\mathrm{MB}$ (CK-MB), 5,38 ng/dl e 8,79 ng/dl (normal < 5,0 ng/dl) -, coletados na chegada e 6 horas após. Foi feito diagnóstico de infarto agudo do miocárdio sem supradesnivelamento do segmento ST. Foi iniciado tratamento com ácido acetilsalicílico 300 mg, clopidogrel 300 mg (dose de ataque), enoxaparina $60 \mathrm{mg}$ duas vezes por dia, nitroglicerina endovenosa, oxigênio e morfina pela intensidade da dor. Seguindo uma estratégia invasiva precoce, em decorrência da permanência da dor torácica, a paciente foi submetida a coronariografia, que evidenciou artérias coronárias sem lesões obstrutivas e com acinesia ântero-apical (Figura 2).

Foi corrigido o diagnóstico inicial para cardiomiopatia induzida por estresse e iniciado o tratamento com diurético, enalapril e metoprolol associado a ansiolítico. A paciente evoluiu satisfatoriamente nas 24 horas subsequentes, sendo encaminhada ao setor de métodos não-invasivos para realização de ecocardiografia transtorácica, que evidenciou disfunção ventricular esquerda significativa, fração de ejeção de $31 \%$, diâmetros diastólico e sistólico aumentados, hipocinesia septo-apical, ântero-apical, látero-apical, ínfero-apical e póstero-apical (Figura 3).

A paciente evoluiu clinicamente bem e realizou nova ecocardiografia transtorácica de controle em seis dias, que evidenciou normalização da função ventricular esquerda (Figura 4).

\section{DISCUSSÃO}

A doença arterial coronária é muito prevalente no Brasil, apresentando elevada morbidade e mortalidade quando se refere ao infarto agudo do miocárdio. Mudanças no estilo de vida acumuladas nas últimas décadas têm aumentado o risco cardiovascular das mulheres e, consequentemente, o risco de infarto agudo do miocárdio. O risco cardiovascular também aumenta após o diagnóstico de infarto agudo do miocárdio. Assim, é importante diferenciá-lo da cardiomiopatia induzida por estresse, que, apesar de ter quadro clínico similar ao do infarto agudo do miocárdio, não aumenta a morbidade e mortalidade a longo prazo.
A cardiomiopatia induzida por estresse é uma síndrome que foi descoberta recentemente e não existem muitas informações sobre sua prevalência. A partir de 2000, vários relatos e série de casos têm descrito essa condição que simula o infarto agudo do miocár$\operatorname{dio}^{4,8,9}$. A prevalência estimada de $1 \%$ a $2 \%$ é proveniente de pacientes com suspeita de síndrome coronária aguda, que foram originados de pequenas séries de casos e que foram incluídos numa grande revisão sistemática relatada por Gianni et al. ${ }^{10}$ ou de um registro de 3.265 pacientes com síndrome coronária aguda e biomarcadores cardíacos elevados relatado por Kurowski et al. ${ }^{6}$. É mais prevalente em mulheres que em homens (aproximadamente 90\%), exatamente o oposto do que ocorre na prevalência da doença arterial coronáriaa ${ }^{2,4,5,7}$. Há relatos de que mulheres na pós-menopausa apresentam maior risco de desenvolver essa cardiomiopatia, com média de idade entre 62 e 75 anos, sendo esse risco de menos de $3 \%$ em mulheres com idade inferior a 50 anos $^{2,7}$. Até o momento, desconhece-se por que essa condição afeta predominantemente as mulheres.

Há incertezas quanto à fisiopatologia dessa doença, porém acredita-se que possa ocorrer disfunção ou espasmo microvascular difuso induzido pela excessiva liberação de catecolaminas desencadeada por estresse físico ou emocional ou por toxicidade direta das catecolaminas no músculo cardíaco, resultando em atordoamento miocárdico ${ }^{3,10-12}$. Frequentemente são identificados fatores de estresse físico ou emocional associados, como morte inesperada de familiares ou amigos, abuso doméstico, catástrofe natural, perda de dinheiro ou falência, falar em público, e acidente de carro $^{4,7,9,13}$. Tsuchihashi et al. ${ }^{4}$ identificaram associação de $20 \%$ com os fatores psicológicos e a maioria dos pacientes $(67 \%)$ iniciou com quadro de dor torácica. Neste caso, a paciente havia sido resgatada de um incêndio quando teve início o quadro de dor torácica.

Não existe consenso quanto aos critérios diagnósticos para cardiomiopatia induzida por estresse. Os sintomas e o eletrocardiograma não têm valor preditivo suficiente para distinguir um paciente com cardiomiopatia induzida por estresse de um com infarto agudo do miocárdio, sendo a angiografia coronária o melhor método diagnóstico isolado para essa condição ${ }^{7,14}$. Dessa forma, em 2004, foram desenvolvidos critérios diagnósticos por pesquisadores da Clínica Mayo, os quais foram modificados recentemente e têm sido comumente utilizados. Devem preencher todos os quatro critérios: 1) hipocinesia ou acinesia transitória do segmento médio do ventrículo esquerdo, com ou sem envolvimento apical (geralmente ocorre redução da fração de ejeção para valores entre $20 \%$ e $49 \%$ ), e anormalidade na contratilidade segmentar, que se estende além da distribuição de um vaso epicárdico, frequentemente associada a um fator estressante; 2) ausência de doença arterial angiográfica significativa ou evidência angiográfica de ruptura de placa; 3) nova 
Busnello R. Cardiomiopatia Induzida por Estresse: Diagnóstico Diferencial de Infarto do Miocárdio nas Emergências. Rev Bras Cardiol Invas. 2009;17(2):274-8.

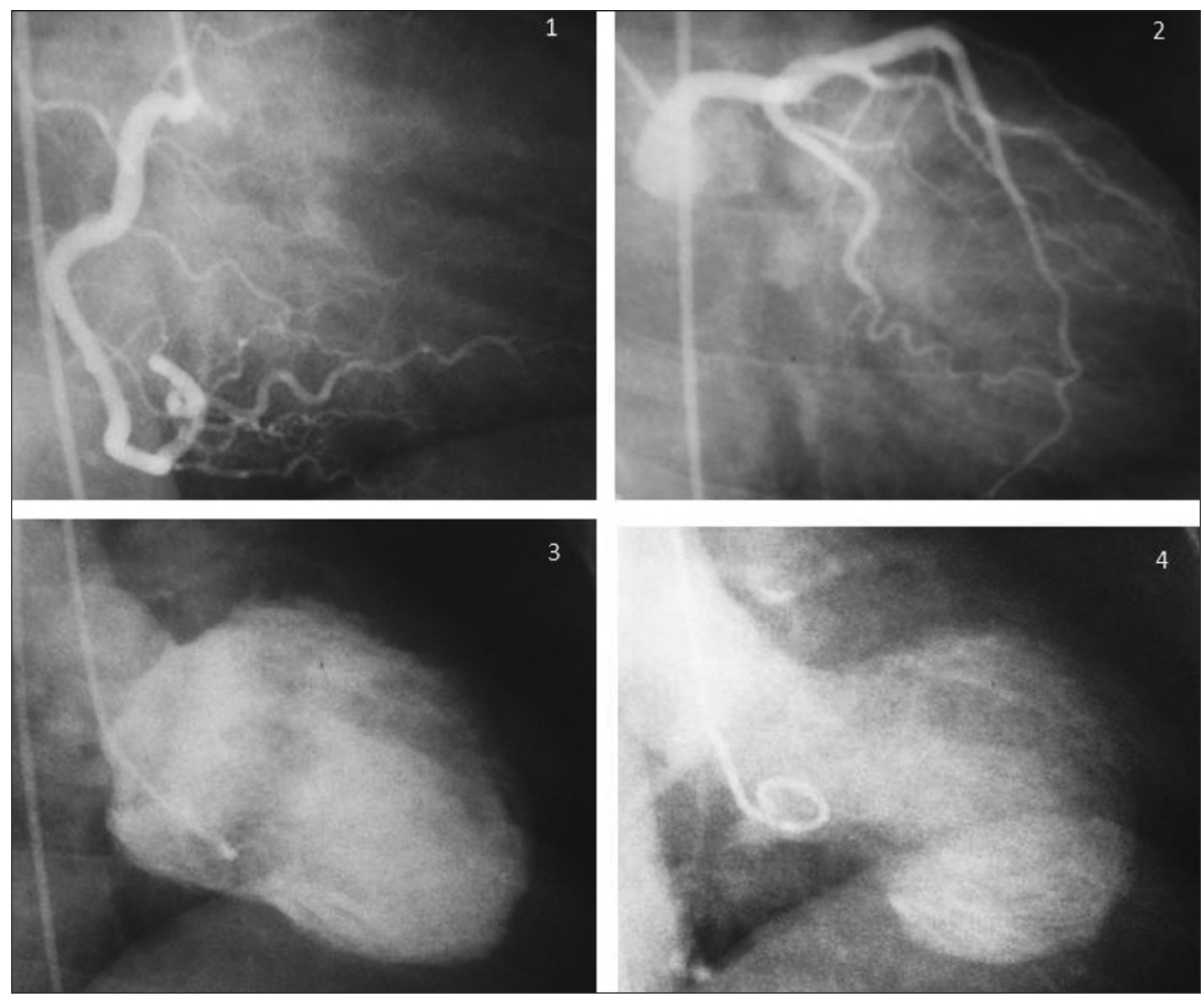

Figura 2 - Cineangiocoronariografia evidenciando: artéria coronária direita dominante (1), sistema coronário esquerdo (2), ventriculografia em fase final da diástole (3), e ventriculografia em fase final da sístole - abaulamento apical do ventrículo esquerdo (4).

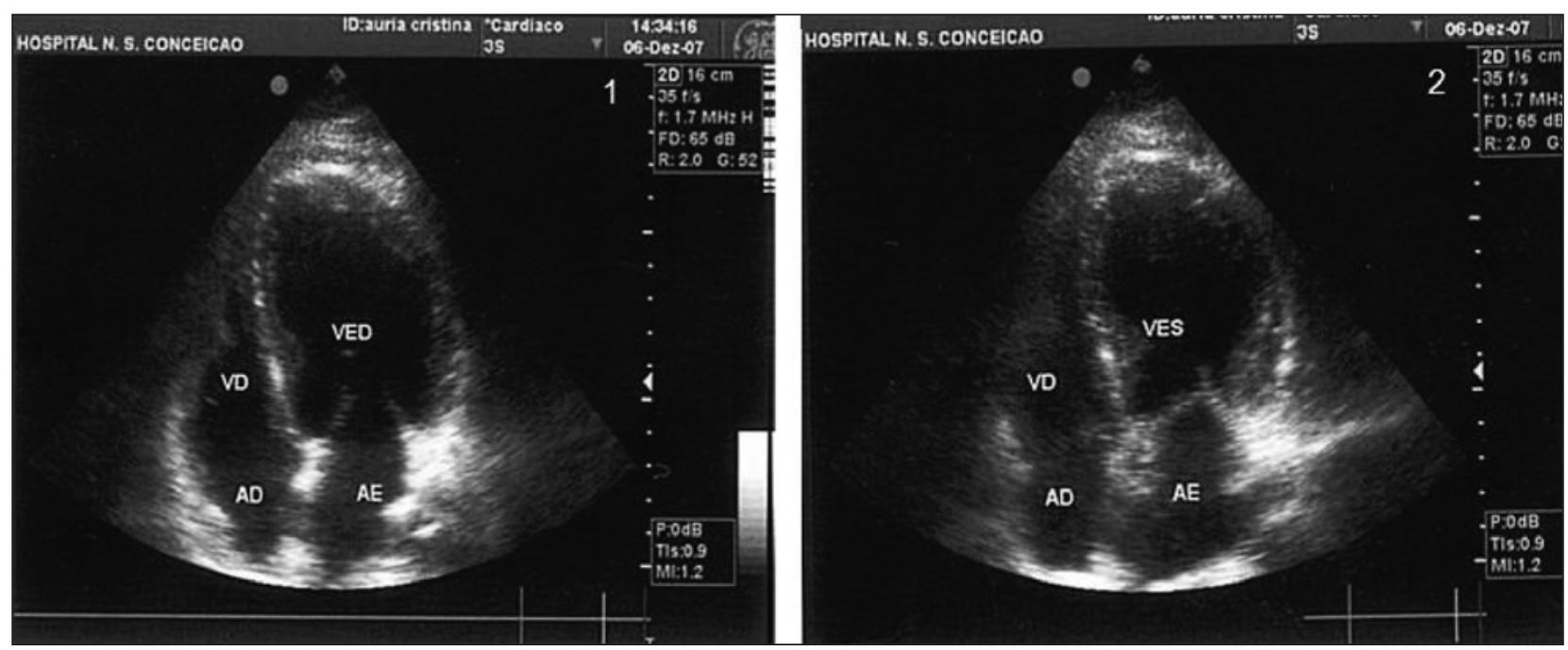

Figura 3 - Ecocardiografia transtorácica: diástole ventricular esquerda (1) e sístole ventricular esquerda com abaulamento apical do ventrículo esquerdo (2). $\mathrm{AD}=$ átrio direito; $\mathrm{AE}=$ átrio esquerdo; $\mathrm{VD}$ = ventrículo direito; $\mathrm{VED}=$ diástole ventricular esquerda; $\mathrm{VES}=$ sístole ventricular esquerda. 
Busnello R. Cardiomiopatia Induzida por Estresse: Diagnóstico Diferencial de Infarto do Miocárdio nas Emergências. Rev Bras Cardiol Invas. 2009;17(2):274-8.

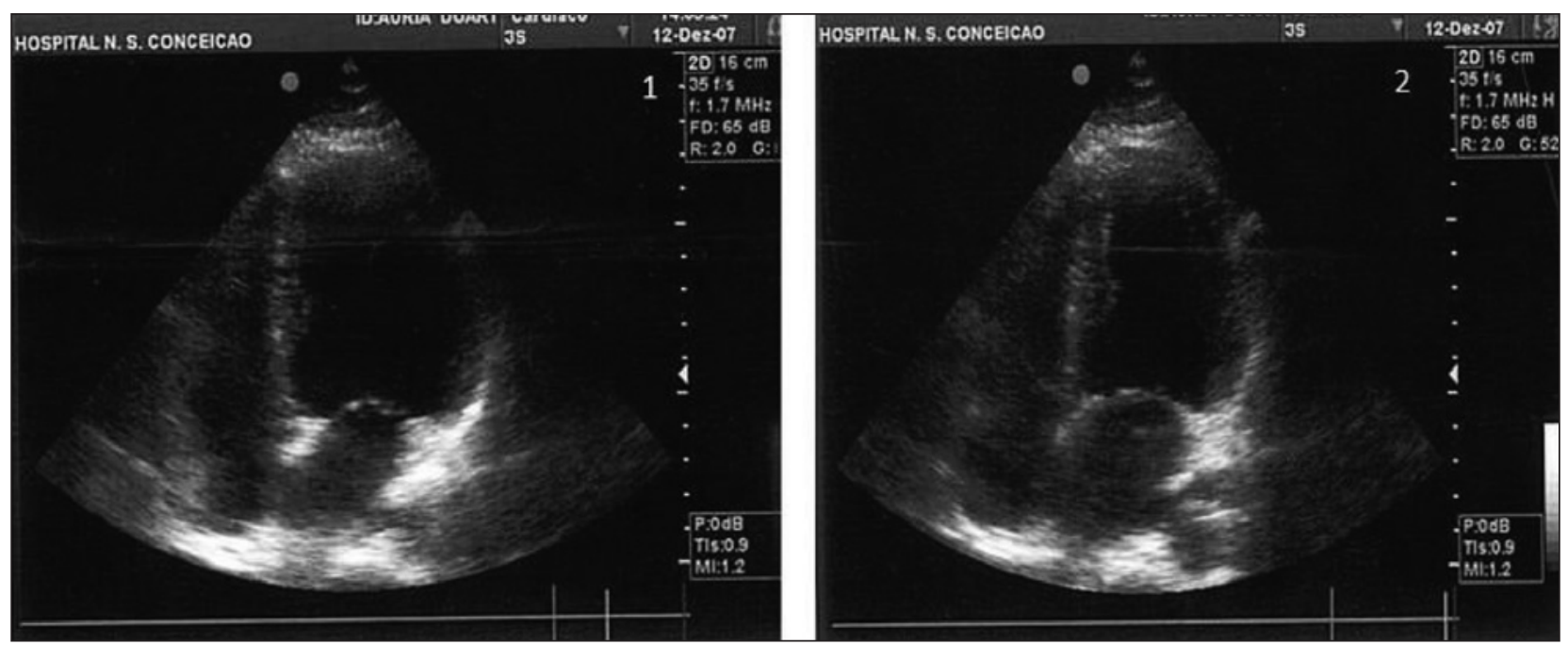

Figura 4 - Ecocardiografia transtorácica: diástole ventricular esquerda (1) e sístole ventricular esquerda com retorno da contratilidade normal (2).

anormalidade eletrocardiográfica (elevação do segmento ST, que é mais freqüente, e cerca de $80 \%$ localizada na parede anterior e/ou inversão da onda T) ou moderada elevação da troponina cardíaca (que frequentemente não se correlaciona com a gravidade do comprometimento hemodinâmico); e 4) ausência de feocromocitoma ou miocardite ${ }^{2,4,5,7,10,13}$. A paciente preenchia todos os critérios descritos.

Não existe tratamento específico para disfunção do ventrículo esquerdo caracterizada pela cardiomiopatia induzida por estresse, visto que a função cardíaca retorna ao normal em algumas semanas. Algumas vezes, a resolução do estresse tanto físico como emocional acarreta rápida melhora dos sintomas e das anormalidades eletrocardiográficas. Por outro lado, pode ser necessário o uso de medicamento e não existem ainda ensaios clínicos para o tratamento dessa síndrome; portanto, podem-se utilizar inibidores da enzima conversora da angiotensina, betabloqueadores e diuréticos se houver sobrecarga de volume ${ }^{2,15}$. Permanece controverso o uso de ácido acetilsalicílico e heparina, em decorrência do risco de ruptura cardíaca associada à forma de balão apical do ventrículo esquerdo. Especialistas sugerem o tratamento com estrógenos para mulheres pós-menopausa que tenham sofrido um episódio de cardiomiopatia induzida por estresse ${ }^{15}$.

O prognóstico da cardiomiopatia induzida por estresse geralmente é favorável, sendo a insuficiência cardíaca, com ou sem edema pulmonar, a complicação mais frequente, presente em aproximadamente $20 \%$ dos $\operatorname{casos}^{7,15}$. A taxa de mortalidade intra-hospitalar varia entre 0 e $8 \%$, apesar de alguns especialistas afirmarem que os dados publicados estão subestima$\operatorname{dos}^{2,4,5,15,16}$. Todos os pacientes que sobrevivem ao episódio agudo recuperam a fração de ejeção entre 1 e 8 semanas ${ }^{4,5,7,8}$. Elesber et al. ${ }^{17}$ evidenciaram taxa de recorrência de aproximadamente $10 \%$ em 4 anos.
O diagnóstico da cardiomiopatia induzida por estresse deve ser de conhecimento de todos os cardiologistas e emergencistas, visto que se trata de uma síndrome recentemente apresentada aos países ocidentais e que apresenta forte associação com situações de estresse, portanto de relevância na atualidade, e cujo tratamento e prognóstico são substancialmente diferentes de um paciente com os mesmos sintomas e infarto agudo do miocárdio.

\section{CONFLITO DE INTERESSES}

Os autores declararam inexistência de conflito de interesses.

\section{REFERÊNCIAS BIBLIOGRÁFICAS}

1. Satoh H, Tateishi H, Uchida T. Takotsubo-type cardiomyopathy due to multivessel spasm. In: Kodama K, Haze K, Hon M, editors. Tokyo: Kagakuhyouronsya; 1990. p.56-64.

2. Bybee KA, Kara T, Prasad A, Lerman A, Barsness GW, Wright RS, et al. Systematic review: transient left ventricular apical ballooning: a syndrome that mimics ST-segment elevation myocardial infarction. Ann Intern Med. 2004;141(11): 858-65.

3. Ako J, Sudhir K, Farouque HM, Honda Y, Fitzgerald PJ. Transient left ventricular dysfunction under severe stress: brain-heart relationship revisited. Am J Med. 2006;119(1): 10-7.

4. Tsuchihashi K, Ueshima K, Uchida T, Oh-mura N, Kimura $\mathrm{K}$, Owa $\mathrm{M}$, et al. Transient left ventricular apical ballooning without coronary artery stenosis: a novel heart syndrome mimicking acute myocardial infarction: Angina PectorisMyocardial Infarction Investigations in Japan. J Am Coll Cardiol. 2001;38(1):11-8.

5. Sharkey SW, Lesser JR, Zenovich AG, Maron MS, Lindberg $\mathrm{J}$, Longe TF, et al. Acute and reversible cardiomyopathy provoked by stress in women from the United States. Circulation. 2005;111(4):472-9.

6. Kurowski V, Kaiser A, Von Hof K, Killermann DP, Mayer B, Hartmann F, et al. Apical and midventricular transient 
Busnello R. Cardiomiopatia Induzida por Estresse: Diagnóstico Diferencial de Infarto do Miocárdio nas Emergências. Rev Bras Cardiol Invas. 2009;17(2):274-8.

left ventricular dysfunction syndrome (tako-tsubo cardiomyopathy): frequency, mechanisms, and prognosis. Chest. 2007; 132(3):809-16.

7. Prasad A, Lerman A, Rihal CS. Apical ballooning syndrome (Tako-Tsubo or stress cardiomyopathy): a mimic of acute myocardial infarction. Am Heart J. 2008;155(3):408-17.

8. Kurisu S, Sato H, Kawagoe $T$, Ishihara M, Shimatani $Y$, Nishioka K, et al. Tako-tsubo-like left ventricular dysfunction with ST-segment elevation: a novel cardiac syndrome mimicking acute myocardial infarction. Am Heart J. 2002;143(3): 448-55.

9. Sato M, Fujita S, Saito A, Ikeda $Y$, Kitazawa H, Takahashi $M$, et al. Increased incidence of transient left ventricular apical ballooning (so-called "Takotsubo" cardiomyopathy) after the mid-Niigata Prefecture earthquake. Circ J. 2006;70(8): 947-53.

10. Gianni M, Dentali F, Grandi AM, Sumner G, Hiralal R, Lonn E. Apical ballooning syndrome or takotsubo cardiomyopathy: a systematic review. Eur Heart J. 2006;27(13):1523-9.

11. Dhar S, Koul D, Subramanian S, Bakhshi M. Transient apical ballooning: sheep in wolves' garb. Cardiol Rev. 2007; 15(3):150-3.
12. Watanabe $\mathrm{H}$, Kodama $\mathrm{M}$, Okura $\mathrm{Y}$, Aizawa $\mathrm{Y}$, Tanabe $\mathrm{N}$, Chinushi $M$, et al. Impact of earthquakes on Takotsubo cardiomyopathy. JAMA. 2005;294(3):305-7.

13. Bybee KA, Prasad A, Barsness GW, Lerman A, Jaffe AS, Murphy JG, et al. Clinical characteristics and thrombolysis in myocardial infarction frame counts in women with transient left ventricular apical ballooning syndrome. Am J Cardiol. 2004;94(3):343-6.

14. Bybee K, Motiei A, Syed I, Kara T, Prasad A, Lennon RJ, et al. Electrocardiography cannot reliably differentiate transient left ventricular apical ballooning syndrome from anterior ST-segment elevation myocardial infarction. J Electrocardiol. 2007;40(1):38.e1-6.

15. Akashi Y, Goldstein D, Barbaro G, Ueyama T. Takotsubo cardiomyopathy: a new form of acute, reversible heart failure. Circulation. 2008;118(25):2754-62.

16. Akashi YJ, Musha H, Kida K, Itoh K, Inoue K, Kawasaki K, et al. Reversible ventricular dysfunction takotsubo cardiomyopathy. Eur J Heart Fail. 2005;7(7):1171-6.

17. Elesber AA, Prasad A, Lennon RJ, Wright RS, Lerman A, Rihal CS. Four-year recurrence rate and prognosis of the apical ballooning syndrome. J Am Coll Cardiol. 2007;50(5): 448-52. 